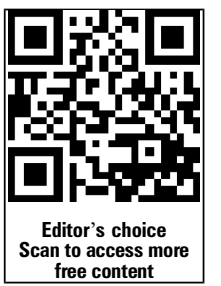

- Additional material is published online only. To view please visit the journal online (http://dx.doi.org/10.1136/ oemed-2013-101997)

${ }^{1}$ Faculty of Health Sciences, University of Sydney, Lidcombe, New South Wales, Australia ${ }^{2}$ Discipline of Physiotherapy, Ageing, Work and Health Research Unit, Faculty of Health Sciences, University of Sydney, Lidcombe, New South Wales, Australia

${ }^{3}$ The George Institute for Global Health, Camperdown, New South Wales, Australia ${ }^{4}$ Discipline of Physiotherapy, Clinical and Rehabilitation Sciences Research Group, Faculty of Health Sciences, University of Sydney, Lidcombe, New South Wales, Australia

\section{Correspondence to} Maria Agaliotis, Faculty of Health Sciences, University of Sydney, 75 East Street, Lidcombe, NSW 1825 Australia;

maria.agaliotis@sydney.edu.au

Received 23 November 2013 Revised 29 March 2014 Accepted 3 May 2014 Published Online First 28 May 2014

\title{
Burden of reduced work productivity among people with chronic knee pain: a systematic review
}

\author{
Maria Agaliotis, ${ }^{1}$ Martin G Mackey, ${ }^{2}$ Stephen Jan, ${ }^{3}$ Marlene Fransen ${ }^{4}$
}

\section{ABSTRACT}

Objective The aims of this systematic review were to determine the prevalence of reduced work productivity among people with chronic knee pain as well as specifically categorise determinants of work productivity losses into individual, disease and work-related factors, conduct an evaluation of study methodological quality and present a best-evidence synthesis.

Methods We searched the literature using combinations of key words such as knee pain, knee osteoarthritis, absenteeism (days taken off work) and presenteeism (reduced productivity while at work) for observational studies published in English.

Methodological quality appraisal and a best-evidence synthesis were used to pool the study findings.

Results The studies were conducted exclusively in high income countries of North America, Western Europe and Hong Kong. 17 studies were included in the review, 10 measuring absenteeism and six measuring presenteeism. Of the 10 studies reporting absenteeism, seven found a 12-month absenteeism prevalence ranging from $5 \%$ to $22 \%$. Only two studies evaluated presenteeism prevalence and reported a range from $66 \%$ to $71 \%$. Using best-evidence synthesis: three high quality cohort studies and three cross-sectional studies provided strong evidence that knee pain or knee osteoarthritis was associated with absenteeism; two high quality crosssectional studies and one cohort study provided limited evidence for an association with presenteeism; one cross-sectional study provided limited evidence for an association among age, high job demands and low coworker support and absenteeism among nurses with knee pain. No studies examined individual or workrelated factors associated with presenteeism.

Conclusions A number of high quality studies consistently demonstrated that chronic knee pain or knee osteoarthritis is associated with absenteeism. However, data are lacking regarding presenteeism and individual or work-related risk factors for reduced work productivity among older workers with chronic knee pain.

Systematic review registration number PROSPERO registry number: CRD42013004137.

Chronic knee pain due to knee osteoarthritis is a common condition among older people, and particularly people aged 50-69 years. ${ }^{12}$ Loss of knee joint function from this condition can potentially cause reduced work productivity. ${ }^{3}$ Moreover, work requirements aggravate knee pain and even the ability to continue working, resulting in forced unemployment or early retirement. ${ }^{4}$ Globally, with an ageing population, an increase in obesity and a tendency to delay retirement, the

\section{What is already known on this topic}

- Chronic knee pain is highly prevalent among people aged 50 years and over in the workforce.

- Occupational risk factors for developing chronic knee pain are well known, but the ongoing consequence of working with chronic knee pain has only recently been explored.

- With an ageing population worldwide, an increase in obesity and a growing tendency to delay retirement, the absolute numbers and relative prevalence of people affected by chronic knee pain in the workforce will increase.

\section{What this paper adds}

- This study provides an updated comprehensive and systematic review of the literature examining absenteeism and presenteeism among people with chronic knee pain, including an evaluation of study methodological quality and a best-evidence synthesis.

- Best-evidence synthesis provides consistent evidence across a number of high quality studies supporting the association of chronic knee pain with absenteeism but limited evidence for an association with presenteeism.

- Due to a paucity of studies, there is limited evidence that individual or work-related factors, such as age, high job demands or low coworker support, are associated with absenteeism among people with chronic knee pain.

- No studies evaluated individual or work-related factors for presenteeism among people with chronic knee pain.
To cite: Agaliotis $\mathrm{M}$ Mackey MG, Jan $\mathrm{S}$, et al. Occup Environ Med 2014;71:651-659 absolute numbers and relative prevalence of people affected by chronic knee pain in the workforce will increase. ${ }^{1}$

Reduced work productivity is typically measured in two ways: as days taken off work (absenteeism) or as self-reported reduced work productivity while at work (presenteeism).

Of late, presenteeism is increasingly recognised as a major contributor to reduce work productivity; however, there is no consensus about a common 
measurement tool or metrics to measure the phenomenon more accurately. ${ }^{5}$

The relationship between work productivity and chronic knee pain has been examined in a number of studies. A large community-based survey has reported the prevalence of knee pain among people aged $40-80$ years to be around $30 \%$, with a higher prevalence demonstrated for certain occupational groups. ${ }^{6}$ There is evidence from population-based surveys in Scandinavia suggesting a diagnosis of knee osteoarthritis ${ }^{7}$ is associated with increased absenteeism. However, a recent review ${ }^{8}$ found patients with hip or knee osteoarthritis were more likely to report reduced work capacity than the general population. A recent longitudinal study among people with chronic knee pain found that absenteeism was related to low psychological wellbeing, whereas presenteeism was associated with poor selfreported physical function and higher occupational physical demands. ${ }^{9}$ There has been a growing interest in conceptualising and adequately measuring the burden of reduced work productivity among people with painful knee osteoarthritis. ${ }^{10} 11$

The most recent systematic review of observational studies in this field has concluded that the effects of chronic knee pain on work productivity are mild. ${ }^{8}$ The aims of the current systematic review are to provide an update as well as specifically categorise determinants of work productivity losses into individual, disease and work-related factors, conduct an evaluation of study methodological quality and present a best-evidence synthesis.

\section{METHODS}

\section{Literature search}

Literature search strategies were developed using medical subjects heading $(\mathrm{MeSH})$ terms and text words related to absenteeism and presenteeism among persons with chronic knee pain (see online supplementary appendix 1). A search of electronic databases was conducted in May 2013. The database searches included MEDLINE (OVID interface, URL 1946 onwards), PsycINFO (OVID interface, 1806 onwards), EMBASE (OVID interface, 1946 onwards) and CINAHL (EBSCO interface, 1981 onwards) (see online supplementary appendix 1). Articles were limited to English language. The references of relevant articles were hand searched. A systematic review protocol was developed according to the PRISMA and STROBE guidelines ${ }^{12} 13$ and registered (PROSPERO registry number: CRD42013004137).

\section{Study selection}

Observational studies that included participants with knee pain and collected data on work participation were included in this review. Chronic knee pain or symptomatic knee osteoarthritis could be self-reported, clinically or radiographically determined, or physician diagnosed. Furthermore, studies must have reported at least one of the following outcomes: absenteeism or presenteeism. Absenteeism was defined as taking a full day or part of the day off work. Presenteeism was defined as being at work but unable to perform at a personal optimal level.

\section{Data extraction}

Titles and abstracts obtained from the literature search were screened for potential inclusion by three reviewers (MA, MF and MGM). Full paper copies of potential relevant papers were retrieved and their reference lists screened. Disagreement or ambiguities were resolved by consensus, after discussion with a fourth reviewer (SJ).

The data extracted included: (1) study characteristics: country, study design, sample size and setting; (2) participant characteristics: age, gender, diagnosis and joint(s) affected, proportion of respondents/participants reporting knee pain or knee osteoarthritis; and (3) absenteeism or presenteeism: definition of work productivity loss (including recall periods), reported prevalence or associations related to absenteeism or presenteeism. Data were extracted by one reviewer (MA) and independently audited by a second (MF).

\section{Data analysis}

Descriptive data tables were developed to explore heterogeneity in study design and definitions of absenteeism and presenteeism. Analysed associations, determinants or risk factors of absenteeism or presenteeism were categorised into individual, disease-related or work-related factors.

\section{Methodological quality assessment}

Three reviewers (SJ with MF or MGM) appraised the methodological quality of each included study using a constructed checklist ${ }^{14}$ based on the recommendations by Sanderson et al. ${ }^{15}$ The 10 -item checklist rated studies on items of internal validity and transparency including appropriate methods of selecting participants, measuring exposure and outcome variables. Each item was allocated a score of either 0 (No or Unsure) or 1 (Yes). In case of disagreement between reviewers, consensus was resolved by a third reviewer (MA). Each study was therefore assigned a score ranging from 0 to 10 . A study was considered to be of high quality if the methodological quality score was $\geq 6^{16}$ (see online supplementary appendix 2).

\section{Best-evidence synthesis}

We used a best-evidence synthesis to summarise the review findings into five levels of evidence (strong, moderate, limited, conflicting or no evidence) based on the criteria set out by Lievense et $a l^{1617}$ (see online supplementary appendix 3).

\section{RESULTS}

In all, 327 references were identified by the literature search (figure 1). Title and abstract screening resulted in 74 full-text articles further assessed for eligibility. Nineteen studies met the inclusion criteria. Bieleman et al ${ }^{18}{ }^{19}$ reported data from the same study population in two published papers: one providing baseline data from a cross-sectional survey, the other a 2-year follow-up longitudinal study. Andersen et $a l^{20} 21$ reported on the same cohort population but at different follow-up periods, one at 3 months and the other after 1 year. The last published paper from each study was included in the review, resulting in 17 original included studies (figure 1).

A descriptive overview of the selected studies examining absenteeism and presenteeism is presented in tables 1 and 2, respectively. Studies evaluating risk factors or determinants associated with absenteeism and presenteeism are presented in tables 3 and 4 , respectively.

\section{Study populations}

Cohort size ranged from $254^{22}$ to 804711 (tables 1 and 2). ${ }^{23}$ Twelve studies (71\%) evaluated subjects with a self-diagnosis of knee osteoarthritis ${ }^{78}$ 24-26 or knee pain ${ }^{20}$ 27-32 while five studies (29\%) confirmed the presence of knee osteoarthritis by a clinical diagnosis. $^{22} 23 \quad 33-35$ Seven studies $(47 \%)^{7} 23242932 \quad 33 \quad 35$ conducted population-based surveys or used population registries, while five studies (29\%) ${ }^{1822} 252634$ recruited participants from clinics and five studies (29\%) were occupation-specific cohorts. $^{2027283031}$ 
Figure 1 Identification and selection of studies.

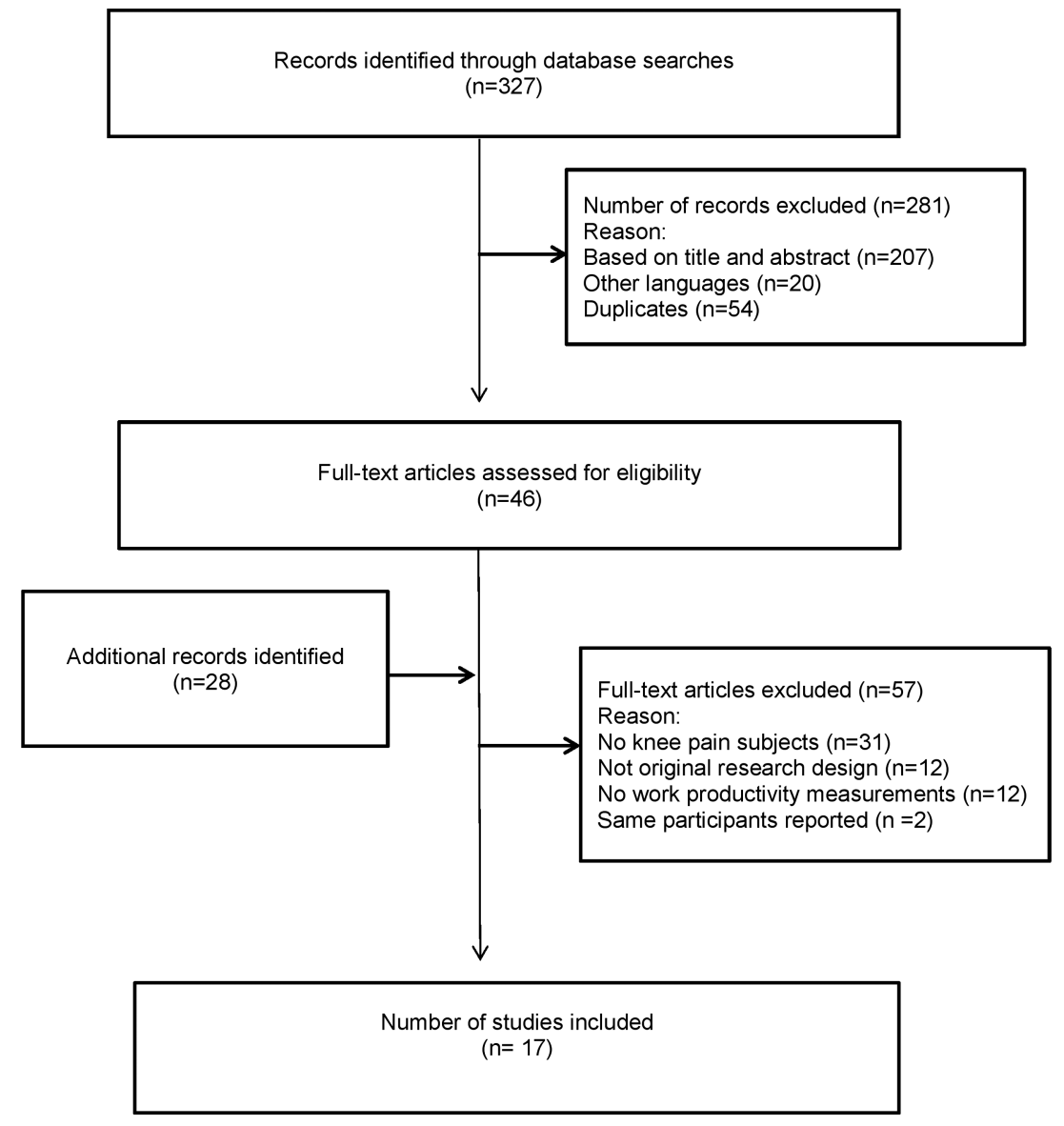

\section{Study design}

There were 10 (59\%) cross-sectional studies $\begin{array}{llllll}24 & 25 & 27 & 29-33 & 35\end{array}$ and $5(29 \%)$ prospective cohort studies: three with a 12 -month follow-up, ${ }^{20} 2328$ one with a 2 -year follow-up ${ }^{18}$ and the other with a 12 -week follow-up. ${ }^{34}$ The other $2(12 \%)$ studies were retrospective in design. ${ }^{22} 26$ In all, 8 (47\%) studies were pub-

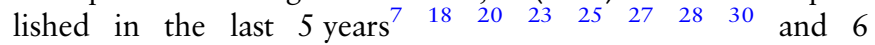
$(35 \%)^{22} 2631-345-10$ years ago and $3(18 \%)^{24} 2935$ more than 10 years ago.

\section{Prevalence of absenteeism}

Overall, $10(59 \%)^{18} \quad 22 \quad 23 \quad 25-27^{29} \quad 32-34$ of the 17 included studies evaluated the prevalence of sick leave or work absenteeism, while 4 (24\%) studies reported the average number of sick leave days (table 1). ${ }^{22} 232634$ Absenteeism was obtained from self-constructed questionnaires in eight studies, ${ }^{22} 25-272932-34$

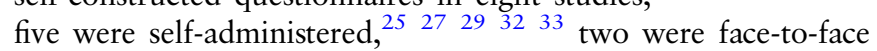
interviews $^{22} 26$ and one conducted weekly telephone interviews. ${ }^{34}$ One study used a validated self-administered questionnaire, ${ }^{18}$ while the other relied on a sickness benefit register. ${ }^{23}$ Sick leave attribution varied and included joint 'pain' ${ }^{25}$ to specific 'knee osteoarthritis'. ${ }^{22}$ Recall periods of absenteeism from work ranged from 'ever', 2933 'in the last week' ${ }^{34}$ to 'in the last 12-months'. $182022 \quad 2325-273132$ Of the 10 studies examining absenteeism prevalence, seven studies had a comparable 12-month recall period and reported an absenteeism prevalence ranging from $5 \%$ to $22 \% .^{18} 22 \quad 2325-2732$ With respect to the remaining three studies: one clinic-based survey collected absenteeism pertaining to the past week and found a high proportion of participants $(33 \%)$ reported 'missed all or part of a day of work/normal activity' due to 'hip or knee pain'; 34 another clinic-based survey found a high proportion (71\%) of participants reporting 'reduced' work hours in the past 12 months because of 'osteoarthritis'; ${ }^{25}$ the third study, a nationwide survey, found $21 \%$ of people with knee osteoarthritis reported 'missed work' ever because of osteoarthritis. ${ }^{33}$ Of the four studies $^{22} 232634$ calculating a mean number of sick days due to knee pain or knee osteoarthritis, three found a range from 3 to 25 days per year. The fourth study found a mean of 0.5 days absent due to knee pain within the last week. ${ }^{34}$

\section{Prevalence of presenteeism}

Only $6(36 \%)$ of the 17 included studies reported a measure of presenteeism (table 2). ${ }^{22} 24 \quad 30 \quad 33-35$ Three studies used varied definitions of presenteeism including 'limitations in the workplace' from knee osteoarthritis ${ }^{33}$ or 'reduced working capacity'. ${ }^{24}$ The remaining two studies used validated self-report (psychometric) measures of presenteeism. ${ }^{30}{ }^{35}$ Lerner et al ${ }^{35}$ developed and validated the Work Limitation Questionnaire, using four scales to assess limitations in performing job demands, each with a score range of 0 ('no limitations') to 100 ('most limitations'). Merrill et $a l^{30}$ used a combination of the Health and Work Performance ${ }^{36}$ and the Work Productivity and Activity Limitations Questionnaires ${ }^{37}$ to develop a 12-item Presenteeism Index Score. The proportion and risk of being in the poorest quintile of presenteeism (greatest loss of work productivity) were evaluated among those with knee or leg pain. The reported reason for presenteeism varied between studies: from the specific, in relation to 'knee osteoarthritis' ${ }^{22}$ to the general, for 'any health reasons or problems'. ${ }^{24} 35$ Of the six studies, ${ }^{22} 24 \quad 3033-35$ two published the percentage of workers experiencing presenteeism, according to the authors' definition 


\begin{tabular}{|c|c|c|c|c|c|c|c|}
\hline Author year country & $\begin{array}{l}\text { Design } \\
\text { Data }\end{array}$ & Study diagnosis and population & $\mathrm{N}$ (\% female) & $\begin{array}{l}\text { Age (years) } \\
\text { mean } \\
\text { (range) }\end{array}$ & $\begin{array}{l}\text { Knee } \\
\text { n (\%) } \\
\text { working \% }\end{array}$ & $\begin{array}{l}\text { Definition } \\
\text { timeframe }\end{array}$ & $\begin{array}{l}\text { Prevalence } \\
\mathrm{n}(\%)\end{array}$ \\
\hline $\begin{array}{l}\text { Alexopoulos } 2011^{27} \\
\text { Greece }\end{array}$ & $\begin{array}{l}\text { Survey } \\
\text { Q }\end{array}$ & $\begin{array}{l}\text { Self-reported } \\
\text { Knee } \\
\text { Nurses }\end{array}$ & $350(72 \%)$ & 38 & $\begin{array}{l}81(23 \%) \\
100 \%\end{array}$ & $\begin{array}{l}\text { Absenteeism } \\
\text { Due to knee pain } \\
\text { Past } 12 \text { months }\end{array}$ & $19(5 \%)$ \\
\hline $\begin{array}{l}\text { Andersen } 2012^{20} \\
\text { Denmark }\end{array}$ & $\begin{array}{l}\text { Cohort } \\
R \& Q\end{array}$ & $\begin{array}{l}\text { Self-reported } \\
\text { All joints } \\
\text { Healthcare workers care }\end{array}$ & 8952 (98\%) & $45(\geq 18)$ & $\begin{array}{l}3070(34 \%) \\
100 \%\end{array}$ & $\begin{array}{l}\text { LTSA } \geq 8 \text { weeks } \\
\text { Any reason } \\
\text { Past } 12 \text { months }\end{array}$ & Not given \\
\hline $\begin{array}{l}\text { Andersen } 2011^{28} \\
\text { Denmark }\end{array}$ & $\begin{array}{l}\text { Cohort } \\
\mathrm{R} \& \mathrm{I}\end{array}$ & $\begin{array}{l}\text { Self-reported } \\
\text { All joints } \\
\text { Blue/white collar workers }\end{array}$ & $\begin{array}{l}5096 \\
(49 \%)\end{array}$ & $40(18-64)$ & $\begin{array}{l}\text { Blue collar workers } 815(16 \%) \\
\text { White collar workers } \\
612(12 \%) \\
100 \%\end{array}$ & $\begin{array}{l}\text { LTSA } \geq 3 \text { weeks } \\
\text { Any reason } \\
\text { Past } 2 \text { years }\end{array}$ & Not given \\
\hline $\begin{array}{l}\text { Bieleman } 2013^{18} \\
\text { Netherlands }\end{array}$ & $\begin{array}{l}\text { Cohort } \\
Q\end{array}$ & $\begin{array}{l}\text { Self-reported } \\
\text { Hip and/or knee } \\
\text { Clinic }\end{array}$ & $925(79 \%)$ & $58(45-65)$ & $379(41 \%) 46 \%$ & $\begin{array}{l}\text { Sick leave } \\
\text { Due to hip/knee complaints } \\
\text { Past } 12 \text { months }\end{array}$ & $48(11 \%)$ \\
\hline $\begin{array}{l}\text { Fautrel } 2005^{33} \\
\text { France }\end{array}$ & $\begin{array}{l}\text { Survey } \\
\text { Q }\end{array}$ & $\begin{array}{l}\text { Diagnosis } \\
\text { All joints } \\
\text { Clinic vs population controls }\end{array}$ & $\begin{array}{l}10295 \text { vs } 16924 \\
(66 \%)\end{array}$ & $67(40-90)$ & 3247 (32\%) 17\% & $\begin{array}{l}\text { Missed workday } \\
\text { Due to OA } \\
\text { Ever }\end{array}$ & $\begin{array}{l}\text { Knee OA: } 115(20.5 \%) \\
\text { Controls: not given }\end{array}$ \\
\hline $\begin{array}{l}\text { Grotle } 2008^{7} \\
\text { Norway }\end{array}$ & $\begin{array}{l}\text { Survey } \\
\text { Q }\end{array}$ & $\begin{array}{l}\text { Self-reported } \\
\text { All joints } \\
\text { Population }\end{array}$ & $\begin{array}{l}3266 \\
(55 \%)\end{array}$ & $\begin{array}{l}\text { Median } 45 \\
(24-76)\end{array}$ & $\begin{array}{l}233(7 \%) \\
71 \% \text { (All OA) }\end{array}$ & $\begin{array}{l}\text { Sick leave } \\
(1-8, \geq 8 \text { weeks }) \\
\text { Any reason } \\
\text { Past } 12 \text { months }\end{array}$ & Not given \\
\hline $\begin{array}{l}\text { Holmberg } 2002^{29} \\
\text { Sweden }\end{array}$ & $\begin{array}{l}\text { Survey } \\
Q\end{array}$ & $\begin{array}{l}\text { Self-reported } \\
\text { All joints } \\
\text { Population } \\
\text { (Famers vs } \\
\text { non-farmers) }\end{array}$ & $\begin{array}{l}1013 \text { vs } 769 \\
(0 \%)\end{array}$ & $50(40-59)$ & $\begin{array}{l}\text { Famers } \\
471(47 \%) \\
\text { Non-farmers } \\
338(44 \%) \\
100 \%\end{array}$ & $\begin{array}{l}\text { Sick-listed } \\
\text { Due to knee problems } \\
\text { Ever }\end{array}$ & $\begin{array}{l}\text { Farmers141 (14\%) } \\
\text { Non-farmers } 138(18 \%)\end{array}$ \\
\hline $\begin{array}{l}\text { Hubertsson } 2013^{23} \\
\text { Sweden }\end{array}$ & $\begin{array}{l}\text { Cohort } \\
\mathrm{R}\end{array}$ & $\begin{array}{l}\text { Diagnosis } \\
\text { Knee } \\
\text { Knee vs general population }\end{array}$ & $\begin{array}{l}15345 \text { vs } 789366 \\
(50 \%)\end{array}$ & $\sim 54(16-64)$ & $\begin{array}{l}15345(2 \%) \\
100 \% \text { working age }\end{array}$ & $\begin{array}{l}\text { Sick days } \\
\text { Any reason } \\
\text { Past } 12 \text { months }\end{array}$ & $\begin{array}{l}\text { Knee OA } 19 \% \\
\text { Population } 8 \%\end{array}$ \\
\hline $\begin{array}{l}\text { Hutchings } 2007^{34} \\
\text { USA }\end{array}$ & $\begin{array}{l}\text { Cohort } \\
1\end{array}$ & $\begin{array}{l}\text { Diagnosis } \\
\text { Knee or hip } \\
\text { Clinic }\end{array}$ & $287(70 \%)$ & $65(50 \geq 80)$ & $\begin{array}{l}235(82 \%) \\
16 \%\end{array}$ & $\begin{array}{l}\text { Missed all/part day work/normal activity } \\
\text { Due to pain } \\
\text { Last week }\end{array}$ & $33 \%$ \\
\hline $\begin{array}{l}\text { Leardini } 2004^{22} \\
\text { Italy }\end{array}$ & $\begin{array}{l}\text { Cohort } \\
Q\end{array}$ & $\begin{array}{l}\text { Diagnosis } \\
\text { Knee } \\
\text { Clinic }\end{array}$ & $254(76 \%)$ & $66(38-89)$ & $\begin{array}{l}254(100 \%) \\
21 \%\end{array}$ & $\begin{array}{l}\text { Lost working days } \\
\text { Due to knee OA } \\
\text { Past } 12 \text { months }\end{array}$ & $22 \%$ \\
\hline $\begin{array}{l}\text { Morken } 2003^{31} \\
\text { Norway }\end{array}$ & $\begin{array}{l}\text { Survey } \\
\text { Q }\end{array}$ & $\begin{array}{l}\text { Self-reported } \\
\text { All joints } \\
\text { Aluminium smelting plants }\end{array}$ & $3036(14 \%)$ & $41(18-64)$ & $316(11 \%) 100 \%$ & $\begin{array}{l}\text { Sickness absence } \\
\text { Due to MSD } \\
\text { Past } 12 \text { months }\end{array}$ & Not given \\
\hline $\begin{array}{l}\text { Picavet } 2003^{32} \\
\text { Netherlands }\end{array}$ & $\begin{array}{l}\text { Survey } \\
\text { Q }\end{array}$ & $\begin{array}{l}\text { Self-reported } \\
\text { All joints } \\
\text { Population }\end{array}$ & $3664(50 \%)$ & $(\geq 25)$ & $\begin{array}{l}802(22 \%) \\
100 \%\end{array}$ & $\begin{array}{l}\text { Sick leave } \\
\text { Due to hip or knee } \\
\text { Past } 12 \text { months: } \\
\leq 1 \text { week } \\
1-4 \text { weeks } \\
\geq 4 \text { weeks }\end{array}$ & $\begin{array}{l}5 \% \\
4 \% \\
4 \%\end{array}$ \\
\hline $\begin{array}{l}\text { Sayre } 2010^{25} \\
\text { Canada }\end{array}$ & $\begin{array}{l}\text { Survey } \\
Q\end{array}$ & $\begin{array}{l}\text { Self-reported } \\
\text { All joints } \\
\text { Clinic }\end{array}$ & $688(60 \%)$ & $62(27-86)$ & $453(66 \%) 100 \%$ & $\begin{array}{l}\text { Reduced hours } \\
\text { Due to } \mathrm{OA} \\
\text { Past } 12 \text { months }\end{array}$ & $57(71 \%)$ \\
\hline $\begin{array}{l}\text { Woo } 2003^{26} \\
\text { Hong Kong }\end{array}$ & $\begin{array}{l}\text { Survey } \\
\mathrm{I}\end{array}$ & $\begin{array}{l}\text { Self-reported } \\
\text { Hip or knee } \\
\text { Clinic }\end{array}$ & $574(76 \%)$ & $(<50-70+)$ & $\begin{array}{l}472(82 \%) \\
19 \%\end{array}$ & $\begin{array}{l}\text { Sick leave } \\
\text { Due to } 0 \mathrm{~A} \\
\text { Past } 12 \text { months }\end{array}$ & $57(10 \%)$ \\
\hline
\end{tabular}

I, interview; LTSA, long-term sickness absenteeism; MSD, musculoskeletal disorders; $O A$, osteoarthritis; $Q$, questionnaire; $R$, register. 
Table 2 Presenteeism among people with knee symptoms

\begin{tabular}{|c|c|c|c|c|c|c|c|}
\hline $\begin{array}{l}\text { Author, year, } \\
\text { country }\end{array}$ & $\begin{array}{l}\text { Design } \\
\text { Data }\end{array}$ & $\begin{array}{l}\text { Study diagnosis } \\
\text { and population }\end{array}$ & N (\% Female) & $\begin{array}{l}\text { Age (years) } \\
\text { Mean } \\
\text { (range) }\end{array}$ & $\begin{array}{l}\text { Knee n (\%) } \\
\text { Working \% }\end{array}$ & $\begin{array}{l}\text { Definition } \\
\text { Timeframe }\end{array}$ & $\begin{array}{l}\text { Prevalence } \\
\text { n (\%) }\end{array}$ \\
\hline $\begin{array}{l}\text { Fautrel } 2005^{33} \\
\text { France }\end{array}$ & $\begin{array}{l}\text { Survey } \\
\text { Q }\end{array}$ & $\begin{array}{l}\text { Diagnosis } \\
\text { All joints } \\
\text { Clinic versus population } \\
\text { controls }\end{array}$ & $\begin{array}{l}10295 \\
\text { vs } 16924 \\
(66 \%)\end{array}$ & $67(40-90)$ & $\begin{array}{l}3247(32 \%) \\
17 \%\end{array}$ & $\begin{array}{l}\text { Limited in your ability } \\
\text { Due to } O A \\
\text { Ever }\end{array}$ & $\begin{array}{l}\text { Knee OA } \\
369(66 \%) \\
\text { Controls } \\
424(14 \%)\end{array}$ \\
\hline $\begin{array}{l}\text { Hutchings } \\
2007^{34} \\
\text { USA }\end{array}$ & $\begin{array}{l}\text { Cohort } \\
\text { I \& D }\end{array}$ & $\begin{array}{l}\text { Diagnosis } \\
\text { Knee or hip } \\
\text { Clinic }\end{array}$ & $287(70 \%)$ & $65(50-\geq 80)$ & $\begin{array}{l}235(82 \%) \\
16 \%\end{array}$ & $\begin{array}{l}\text { Days with limited } \\
\text { productivity } \\
\text { Due to joint pain } \\
\text { Past week }\end{array}$ & Not given \\
\hline $\begin{array}{l}\text { Leardini } 2004^{22} \\
\text { Italy }\end{array}$ & $\begin{array}{l}\text { Cohort } \\
\text { Q }\end{array}$ & $\begin{array}{l}\text { Diagnosis } \\
\text { Knee } \\
\text { Clinic }\end{array}$ & $254(76 \%)$ & 66 (38-89) & $\begin{array}{l}254(100 \%) \\
21 \%\end{array}$ & $\begin{array}{l}\text { Loss of working activity } \\
\text { Due to knee } \mathrm{OA} \\
\text { Past } 12 \text { months }\end{array}$ & 0 \\
\hline $\begin{array}{l}\text { Lerner } 2001^{35} \\
\text { USA }\end{array}$ & $\begin{array}{l}\text { Survey } \\
\text { Q }\end{array}$ & $\begin{array}{l}\text { Diagnosis } \\
\text { All joints } \\
\text { Clinic versus healthy } \\
\text { controls }\end{array}$ & $\begin{array}{l}230 \text { vs } 37 \\
(63 \%)\end{array}$ & $\begin{array}{l}54 \text { vs } 45 \\
(18-65)\end{array}$ & $\begin{array}{l}53(17 \%) \\
100 \%\end{array}$ & $\begin{array}{l}\text { Work Limitations } \\
\text { Questionnaire } \\
\text { (0-100) } \\
\text { Due to health problems } \\
\text { Past } 2 \text { weeks }\end{array}$ & $\begin{array}{l}\text { Demand Score } \chi \text { (SE) } \\
\text { Physical } 25 \text { (3) vs } 5 \text { (1) } \\
\text { Mental18 (4) vs } 10(3) \\
\text { Output } 18 \text { (3) vs } 7(3) \\
\text { Time management } 26 \text { (4) } \\
\text { vs } 7 \text { (3) }\end{array}$ \\
\hline $\begin{array}{l}\text { Makela } 1993^{24} \\
\text { Finland }\end{array}$ & $\begin{array}{l}\text { Survey } \\
\text { Q }\end{array}$ & $\begin{array}{l}\text { Self-reported } \\
\text { All joints } \\
\text { Population }\end{array}$ & 5673 (not given) & $\begin{array}{l}\text { Not given } \\
(30-64)\end{array}$ & $\begin{array}{l}229(4 \%) \\
79 \%\end{array}$ & $\begin{array}{l}\text { Reduced work capacity } \\
\text { For health reasons } \\
\text { Ever }\end{array}$ & $\begin{array}{l}163(71 \%) \text { knee OA vs } \\
1373(24 \%) \text { entire cohort }\end{array}$ \\
\hline $\begin{array}{l}\text { Merrill } 2012^{30} \\
\text { USA }\end{array}$ & $\begin{array}{l}\text { Survey } \\
\text { Q }\end{array}$ & $\begin{array}{l}\text { Self-reported } \\
\text { Knee or leg pain } \\
\text { Three companies }\end{array}$ & $19803(62 \%)$ & $44(18-83)$ & $\begin{array}{l}3700 \text { (approx.) } \\
100 \%\end{array}$ & $\begin{array}{l}\text { Presenteeism Index } \\
\text { Score }(0-100) \\
\text { Due to knee condition } \\
\text { Past } 4 \text { weeks }\end{array}$ & $\begin{array}{l}\text { Poorest quintile } \\
2835(18 \%) \text { no knee pain } \\
1111(30 \%) \text { knee pain }\end{array}$ \\
\hline
\end{tabular}

D, diary; I, interview; OA, osteoarthritis; Q, questionnaire.

of reduced work productivity, either in relation to their overall health or osteoarthritis, ranging from $66 \%$ to $71 \% .^{24} 33$ The third study, a retrospective cohort study, did not find 'lost work activity' related to knee osteoarthritis in the 12 -month period. ${ }^{22}$ In a nationwide survey, Fautrel et al found workers with knee osteoarthritis (clinic-based cases) were more likely to report a limited ability to work (presenteeism) (66\%) when compared with same sex-age working population controls $(14 \%) .{ }^{33}$

\section{Determinants for absenteeism}

Of the 17 included studies, 8 (47\%) explored determinants of absenteeism (table 3). Seven of the eight studies examined

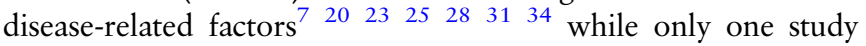
examined possible work-related and individual factors contributing to absenteeism. ${ }^{27}$

Regarding disease-related factors, six of seven studies found a positive association among duration of pain days, ${ }^{20}$ diagnosis of knee osteoarthritis, ${ }^{723}$ presence of knee pain $^{31}$ or increase in pain scores ${ }^{34}$ and increased absenteeism from work. Of these six studies, three were cohort studies and three were crosssectional surveys. One cross-sectional study found an association between knee pain 'often or very often' and increased number of sick leave days ${ }^{31}$ (1-12 days RR 1.8 (95\% CI 1.4 to 2.3) and $>12$ days: RR 2.2 (95\% CI 1.7 to 2.9)). Two studies found those with a diagnosis of knee osteoarthritis were significantly more likely to take days off work compared with those without knee osteoarthritis (RR 1.91 (95\% CI 1.84 to 1.98$)^{23}$ and OR $1.43(95 \% \text { CI } 1.02 \text { to } 2.01)^{25}$ ). A cohort study of healthcare workers in eldercare found a dose-response relationship between duration of knee pain and long-term sickness absence. ${ }^{20}$ However, the same authors found in another study that the risk of long-term sickness absence among blue and white collar workers with severe knee pain was not significantly increased once adjusted through multivariate analysis. ${ }^{28}$ A smaller study of 47 workers found over a 3-month follow-up period that a decrease in joint pain was strongly associated with a decreased number of 'days missed work'. ${ }^{34}$

Work-related factors for absenteeism were assessed in only one of the eight studies. ${ }^{27}$ This cross-sectional study found low coworker support (OR 3.13 (95\% CI 1.12 to 8.78)) and high job demands (OR 4.60 (95\% CI 1.57 to 13.50)) were associated with absenteeism among nursing staff with knee pain. ${ }^{27}$

Finally, only one study evaluated individual factors related to absenteeism and found age was positively associated with an increased risk of absenteeism among nursing staff with knee complaints. Compared with people aged 30 years and below, those aged 45 years and over were approximately 12 times (OR 11.92 (95\% CI 1.44 to 98.81)) more likely associated with absenteeism over the past 12 -months. ${ }^{27}$

\section{Determinants for presenteeism}

Three of six included studies reporting presenteeism as an outcome examined possible determinants of presenteeism (table 4). ${ }^{24} 3034$ Regarding disease-related factors, all three studies found an association among knee pain, ${ }^{30}$ change in pain $^{34}$ or diagnosis of knee osteoarthritis ${ }^{24}$ and presenteeism. One large cross-sectional study conducted among three companies $^{30}$ found participants with knee or leg pain were two times more likely to be in the poorest quintile on the Presenteeism Index Score (OR 1.72 (95\% CI 1.62 to 1.83$)$ ), while a population-based survey ${ }^{24}$ demonstrated knee osteoarthritis was associated reduced work capacity among 30-65 year olds (OR 3.8 (95\% CI 2.8 to 5.2 )). Finally, a prospective study conducted among workers over a 3 -month period showed changes in pain levels from 'unacceptable' to 'acceptable' improved productivity by half-a-day per week. ${ }^{34}$

No studies were found that evaluated individual or workrelated determinants for presenteeism among people with chronic knee pain or knee osteoarthritis. 
Table 3 Determinants of absenteeism

\begin{tabular}{|c|c|c|}
\hline Study & Variable & Effect $(95 \% \mathrm{Cl})$ \\
\hline \multicolumn{3}{|l|}{ Disease-related factors } \\
\hline \multirow[t]{3}{*}{ Andersen et $a{ }^{20}$ Denmark } & No knee pain & 1 \\
\hline & Knee pain $1-30$ days & HR 1.23 (1.00 to 1.51$)$ \\
\hline & Knee pain $\geq 30$ days & HR 2.04 (1.65 to 2.53 ) \\
\hline \multirow[t]{2}{*}{ Andersen et $a l^{28}$ Denmark } & No knee pain & 1 \\
\hline & Knee pain & HR 1.12 (0.92 to 1.37 ) \\
\hline \multirow[t]{3}{*}{ Grotle et $a l^{7}$ Norway } & No knee $0 A /$ no absenteeism & 1 \\
\hline & Knee OA/1-8 weeks absent & OR 2.03 (1.20 to 3.44$)$ \\
\hline & Knee $O A / \geq 8$ weeks absent & OR 1.95 (1.08 to 3.50$)$ \\
\hline \multirow[t]{2}{*}{ Hubertsson et $a l^{23}$ Sweden } & No knee $\mathrm{OA}$ & 1 \\
\hline & Knee OA & RR 1.91 (1.84 to 1.98$)$ \\
\hline Sayre et $a l^{25}$ Canada & Knee OA & OR 1.43 (1.02 to 2.01$)$ \\
\hline Morken et al ${ }^{31}$ Norway & $\begin{array}{l}\text { No knee pain often/very often } \\
\text { Knee pain often/very often }\end{array}$ & $\begin{array}{l}1 \\
\text { STSA: RR } 1.8 \text { (1.4 to } 2.3 \text { ) } \\
\text { LTSA: RR } 2.2 \text { ( } 1.7 \text { to } 2.9 \text { ) }\end{array}$ \\
\hline \multirow[t]{2}{*}{ Hutchings et al ${ }^{34}$ USA } & Pain subscale score $(0-20)$ & 2-unit decrease reduced absenteeism by $48 \%$ \\
\hline & Improvement in pain levels & Decreased the number of days missed work by $64 \%$ \\
\hline \multicolumn{3}{|c|}{ Individual-related factors among people with knee pain } \\
\hline \multirow[t]{2}{*}{ Alexopoulos et al ${ }^{27}$ Greece } & Age $\leq 30$ years & 1 \\
\hline & Age $\geq 45$ years & OR 11.92 (1.44 to 98.81$)$ \\
\hline \multicolumn{3}{|c|}{ Work-related factors among people with knee pain } \\
\hline \multirow[t]{2}{*}{ Alexopoulos et al ${ }^{27}$ Greece } & High coworker support & 1 \\
\hline & Low coworker support & OR 3.13 (1.12 to 8.78$)$ \\
\hline \multirow[t]{2}{*}{ Alexopoulos et al ${ }^{27}$ Greece } & Low job demands & 1 \\
\hline & High job demands & OR 4.60 (1.57 to 13.50$)$ \\
\hline
\end{tabular}

HR, hazards ratio; LTSA, long-term sickness absence; OA, osteoarthritis; OR, odds ratio; RR, relative risk; STSA, short-term sickness absence.

\section{Methodological quality assessment}

Table 5 shows the studies ranked by methodological score, and categorised according to their study design (cohort study or cross-sectional survey). Two independent reviewers scored a total of 170 items and agreed on 144 (85\%) (к 0.70 (95\% CI 0.55 to 0.78$), \mathrm{p}<0.001)$. Most of the disagreements were caused by interpretation of the criteria or unclear reporting in the study for multivariate analysis or specific sources of nonresponse bias. A total of $13(76 \%)$ studies would be considered of high quality (score $\geq 6$ ).

\section{Synthesis of the evidence}

Three cohort $^{20} \quad 23{ }^{34}$ and three cross-sectional ${ }^{7} \quad 2531$ high quality studies provide strong evidence for the association between knee pain or knee osteoarthritis and being absent from work, while two cross-sectional ${ }^{24} 30$ and one cohort ${ }^{34}$ high quality study provide only limited evidence for an association with presenteeism. One high quality cross-sectional study ${ }^{27}$ provides limited evidence for the association among age, high job demands and low coworker support and absenteeism among people with chronic knee pain. No studies examined individual or work-related factors associated with presenteeism among people with chronic knee pain.

\section{DISCUSSION}

The studies included in this systematic review of work productivity among people with chronic knee pain were conducted in 17 distinct population or clinic-based cohorts drawn from 11 countries. This review reveals that the high quality studies conducted in this area consistently demonstrate that chronic knee pain or knee osteoarthritis is an important determinant for

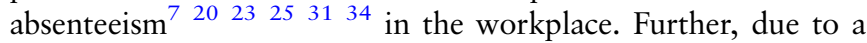
paucity of studies, there is also only limited evidence that individual or work-related factors, such as age, high job demands or low coworker support, are associated with absenteeism among people with chronic knee pain. In contrast, this review could

Table 4 Determinants of presenteeism

\begin{tabular}{|c|c|c|}
\hline Risk-related factor & Variable & Effect $(95 \% \mathrm{Cl})$ \\
\hline \multicolumn{3}{|l|}{ Disease-related factors } \\
\hline Hutchings et $a l^{34}$ USA & Change in pain levels from 'unacceptable' to 'acceptable' & Improved productivity by half-a-day per week \\
\hline \multirow{2}{*}{ Merrill et al ${ }^{30}$ USA } & No knee or leg pain & 1 \\
\hline & Knee or leg pain & OR 1.72 (1.62 to 1.83 ) \\
\hline \multirow[t]{2}{*}{ Makela et $a l^{24}$ Finland } & No knee osteoarthritis & 1 \\
\hline & Knee osteoarthritis & OR 3.8 (2.8 to 5.2 ) \\
\hline
\end{tabular}


Table 5 Methodological quality of studies ${ }^{14}$

\begin{tabular}{|c|c|c|c|c|c|c|c|c|c|c|c|}
\hline Author, year, country & 1 & 2 & 3 & 4 & 5 & 6 & 7 & 8 & 9 & 10 & Score \\
\hline \multicolumn{12}{|l|}{ Cohort } \\
\hline Hutchings et al 2007, USA ${ }^{34}$ & Y & $Y$ & Y & $Y$ & Y & $Y$ & $Y$ & Y & $Y$ & $Y$ & 10 \\
\hline Andersen et al 2012, Denmark ${ }^{20}$ & Y & $\mathrm{N}$ & Y & Y & Y & $\mathrm{N}$ & Y & Y & Y & Y & 8 \\
\hline Andersen et al 2011, Denmark ${ }^{28}$ & Y & $\mathrm{N}$ & Y & Y & Y & $\mathrm{N}$ & $\mathrm{Y}$ & Y & $\mathrm{Y}$ & Y & 8 \\
\hline Hubertsson et al 2013, Sweden ${ }^{23}$ & Y & Y & Y & $\mathrm{N}$ & Y & N & Y & Y & $\mathrm{Y}$ & Y & 8 \\
\hline Bieleman et al 2013, The Netherlands ${ }^{18}$ & $Y$ & $\mathrm{U}$ & Y & $Y$ & $\mathrm{~N}$ & Y & $Y$ & $Y$ & Y & $\mathrm{U}$ & 7 \\
\hline Leardini et al 2004, Italy 22 & $u$ & $\mathrm{U}$ & N & Y & U & $\mathrm{U}$ & $\mathrm{N}$ & $\mathrm{N}$ & $\mathrm{N}$ & $\mathrm{N}$ & 1 \\
\hline \multicolumn{12}{|l|}{ Cross-sectional } \\
\hline Merrill et al 2012, USA ${ }^{30}$ & $Y$ & $Y$ & Y & $Y$ & Y & $Y$ & $Y$ & Y & $Y$ & Y & 10 \\
\hline Holmberg et al 2002, Sweden ${ }^{29}$ & Y & Y & Y & Y & $\mathrm{N}$ & $\mathrm{N}$ & $\mathrm{Y}$ & Y & Y & Y & 8 \\
\hline Picavet and Schouten 2003, The Netherlands ${ }^{32}$ & Y & $\mathrm{N}$ & Y & Y & Y & $\mathrm{N}$ & Y & Y & Y & Y & 8 \\
\hline Grotle et al 2008, Norway ${ }^{7}$ & Y & $\mathrm{N}$ & Y & Y & Y & U & $\mathrm{Y}$ & Y & Y & Y & 7 \\
\hline Makela et al 1993, Finland ${ }^{24}$ & Y & U & Y & Y & U & $u$ & Y & Y & Y & Y & 7 \\
\hline Morken et al 2003, Norway ${ }^{31}$ & $\mathrm{~N}$ & Y & Y & Y & N & $\mathrm{N}$ & $\mathrm{Y}$ & Y & Y & Y & 7 \\
\hline Alexopoulos et al 2011, Greece ${ }^{27}$ & Y & $\mathrm{N}$ & u & Y & $\mathrm{N}$ & $\mathrm{N}$ & $Y$ & Y & $\mathrm{Y}$ & Y & 6 \\
\hline Sayre et al 2010, Canada ${ }^{25}$ & u & $\mathrm{N}$ & Y & Y & $\mathrm{N}$ & u & $Y$ & Y & $\mathrm{Y}$ & Y & 6 \\
\hline Fautrel et al 2005, France ${ }^{33}$ & Y & $\mathrm{N}$ & Y & Y & u & $\mathrm{N}$ & $\mathrm{N}$ & $\mathrm{N}$ & Y & Y & 5 \\
\hline Lerner et al 2001, USA ${ }^{35}$ & Y & U & Y & Y & Y & $\mathrm{N}$ & $\mathrm{N}$ & $\mathrm{N}$ & $\mathrm{N}$ & Y & 5 \\
\hline Woo J et al 2003, Hong Kong ${ }^{26}$ & $u$ & U & Y & Y & U & $\mathrm{N}$ & $Y$ & $Y$ & $\mathrm{~N}$ & $Y$ & 5 \\
\hline
\end{tabular}

$\mathrm{Y}$, yes; $\mathrm{N}$, no; $U$, unsure.

only identify limited evidence for presenteeism. ${ }^{24} \quad 30 \quad 34$ Interestingly, there were no studies evaluating individual or workplace determinants for presenteeism among people with chronic knee pain or knee osteoarthritis.

This systematic review updates and extends a review conducted in $2009^{8}$ by inclusion of a further seven relevant studies, three of which were published in 2012. ${ }^{18} 202325272830$ The current review highlights the heterogeneity of studies examining absenteeism and presenteeism among people with chronic knee pain or knee osteoarthritis. While the overall methodological quality of the studies included in this review was high (table 5), there is still an absolute dearth of studies evaluating risk factors for absenteeism or presenteeism specific to people with lower limb joint osteoarthritis.

The large differences between studies in prevalence estimates of absenteeism and presenteeism are likely to be attributable to differences in study cohorts, recall periods and the specific definition of reduced work productivity used (tables 1 and 2). Studies in this review have largely relied on study-specific questionnaires or surveys when investigating participants' absenteeism or presenteeism. Further, there is large disparity in attribution between studies; participants may be requested to specify work productivity loss or absence as related to their 'knees'22 272930 or 'knee or hips'18 2632 or to relate work loss to 'overall musculoskeletal pain' 31 or 'for any health reason'. ${ }^{24} 35$ Non-specific attribution makes it difficult to get a precise estimate of the burden of absenteeism or presenteeism attributable to chronic knee pain.

There are also inconsistencies with recall periods of absenteeism or presenteeism ranging from 'ever', 2933 or 'in the last 12-months,7 $1820222325-273132$ to 'in the last two weeks'. ${ }^{35}$ The shorter recall period it could be argued would result in a more precise estimate. ${ }^{38}$ However, a single or short period may underestimate disease burden due to the fluctuating symptomatology typical of knee osteoarthritis. Only one study used a methodology of weekly telephone calls over a 3-month period to provide, arguably, a more accurate account of work productivity losses due to chronic knee pain. ${ }^{34}$
It is interesting to note that none of the studies included in this review explored the influence of obesity on the relationship between chronic knee pain and reduced work productivity.

Considering the global need for people to continue working at an older age and increase in obesity, ${ }^{39}$ Outcome Measures in Rheumatology Trials (OMERACT), an independent body of clinicians and researchers, developed validated outcome measurements for osteoarthritis. ${ }^{39}$ Since 2007, OMERACT has established a work productivity group which aims to establish a consensus on accurate and reliable measures of absenteeism and presenteeism. ${ }^{39} 40$ This group has established standardised definitions of absenteeism and presenteeism and recognised various work productivity tools available to date. Furthermore, OMERACT has recommended designing longitudinal studies with a set of standard questionnaires examining absenteeism and presenteeism as well as a range of clinical, psychosocial and socioeconomic risk factors including job satisfaction, job demands, access to work adaptations, physical work demands, psychosocial support and the workplace culture. ${ }^{11} 4041$ All these measures will help identify potentially modifiable risk factors for reduced work productivity and work transitions for this population.

Our systematic review did not examine all indicators of reduced work productivity. For example, three studies included examined work transitions over time such as 'quit job', 26 'change of work' 22 and 'stopped working'18 due to knee problems or change in work behaviour to accommodate knee pain. ${ }^{18}$ In the retrospective cohort study ${ }^{22}$ and clinic-based survey, ${ }^{26}$ between $2 \%$ and $5 \%$ quit their job because of their osteoarthritis. Both studies also found about $2 \%$ changed their jobs either because of osteoarthritis ${ }^{26}$ or for no defined reason. ${ }^{22}$ The 2-year cohort study examined changes in work adaptations over a 2-year period and found unmet (desired) work adaptations (26\%) were higher than actual work adaptations $(20 \%)$ indicating ongoing workplace barriers.

It is apparent from this review that the studies examining determinants of reduced work productivity among people with chronic knee pain were mostly conducted within specific occupations. $^{20} 27283031$ This does not lend to the generalisability of findings, since the occupations were predominately 
women-oriented and of high physical intensity; in this case, healthcare workers ${ }^{20}$ or nurses, ${ }^{27}$ and industrial workers. ${ }^{31}$

A limitation of the findings of this systematic review is that the studies were generally conducted in high income countries that are mostly favourable to income support systems or 'degrees of generosity', ${ }^{42}$ such as paid sick leave. However, even among these countries there are large differences in availability of paid sick leave. The differences include workplace regulations, such as wage replacement or period of sick leave. Globally, accessible sick leave can range from less than 7 days to 2 years. $^{42}$ There are also difference in sick leave definitions; Scandinavian countries often being more generous and transparent in days taken off work. Number of days off work are collected via a registry and depending on the length of time off work are classified as either short-term $(<3-8$ weeks) or longterm (paid) sickness absence. ${ }^{20} 232831$ Not unexpectedly, countries which have more generous provisions tend to report the highest number of absence days.

To conclude, despite a growing number of studies examining the burden of reduced work productivity among people with chronic musculoskeletal conditions, there is little information available examining potentially modifiable individual and workplace risk factors for reduced work productivity specific to people with chronic knee pain. Recommendations for future research would include high quality longitudinal studies measuring absenteeism and presenteeism concurrently, specific to knee problems with shorter recall periods and using standardised validated questionnaires. A longitudinal study design would also allow a documentation of forced work transitions and pain fluctuations. Details of the favourable physical, cultural and psychosocial work environments are required to assist in developing effective individual or workplace strategies for people with chronic knee pain. It is apparent that the prevalence of chronic knee pain in the workforce will increase rapidly over the next decades due to an ageing population and the higher prevalence of obesity in the community. As more people will be required to extend their employment beyond the traditional retirement age, there is a need to develop effective specific workplace strategies to allow people with chronic knee pain to remain active contributors in the workforce.

\section{Acknowledgements This work was supported by Arthritis Australia (The Kevin R} James Grant) awarded to Ms Maria Agaliotis.

Contributors All the authors were involved in acquisition of data; analysis and interpretation of data; drafting the article or revising the paper for important intellectual content; and approved the final version to be published. MA had full access to all of the data in the study and takes full responsibility for the integrity of the data and accuracy of the data analysis. Study concept and design: MA and MF.

Funding Arthritis Australia (The Kevin R James Grant).

Competing interests None.

Provenance and peer review Not commissioned; externally peer reviewed.

\section{REFERENCES}

1 Woolf AD, Pfleger B. Burden of major musculoskeletal conditions. Bull World Health Organ 2003:81:646-56.

2 Busija L, Bridgett L, Williams SRM, et al. Osteoarthritis. Baillieres Best Pract Res Clin Rheumatol 2010;24:757-68.

3 Theis KA, Murphy L, Hootman JM, et al. Prevalence and correlates of arthritis-attributable work limitation in the US population among persons ages 18-64: 2002 National Health Interview Survey Data. Arthritis Rheum 2007; 57:355-63.

4 Miranda $H$, Viikari-Juntura E, Martikainen $\mathrm{R}$, et al. A prospective study on knee pain and its risk factors. Osteoarthritis Cartilage 2002;10:623-30.

5 Schultz $A B$, Edington DW, Schultz $A B$, et al. Employee health and presenteeism: a systematic review. J Occup Rehabil 2007;17:547-79.

6 O'Reilly SC, Muir KR, Doherty M. Occupation and knee pain: a community study. Osteoarthritis Cartilage 2000;8:78-81.
7 Grotle M, Hagen KB, Natvig B, et al. Prevalence and burden of osteoarthritis: results from a population survey in Norway. J Rheumatol 2008;35:677-84.

8 Bieleman HJ, Bierma-Zeinstra SMA, Oosterveld FGJ, et al. The effect of osteoarthritis of the hip or knee on work participation. J Rheumatol 2011;38:1835-43.

9 Agaliotis M, Fransen M, Bridgett L, et al. Risk factors associated with reduced work productivity among people with chronic knee pain. Osteoarthritis Cartilage 2013;21:1160-9.

10 Burton WN, Chen $C Y$, Schultz $A B$, et al. Worker productivity loss associated with arthritis. Dis Manage 2006;9:131-43.

11 Tang K, Escorpizo R, Beaton DE, et al. Measuring the impact of arthritis on worker productivity: perspectives, methodologic issues, and contextual factors. J Rheumatol 2011;38:1776-90.

12 Moher D, Liberati A, Tetzlaff J, et al. Preferred reporting items for systematic reviews and meta-analyses: the PRISMA statement. BMJ 2009;339:b2535.

13 von Elm E, Altman DG, Egger M, et al. The Strengthening the Reporting of Observational Studies in Epidemiology (STROBE) statement: guidelines for reporting observational studies. Lancet 2007;370:1453-7.

14 Lenssinck ML, Burdorf A, Boonen A, et al. Consequences of inflammatory arthritis for workplace productivity loss and sick leave: a systematic review. Ann Rheum Dis 2013;72:493-505.

15 Sanderson S, Tatt ID, Higgins JPT. Tools for assessing quality and susceptibility to bias in observational studies in epidemiology: a systematic review and annotated bibliography. Int J Epidemiol 2007;36:666-76.

16 Lievense AM, Bierma-Zeinstra SM, Verhagen AP, et al. Influence of obesity on the development of osteoarthritis of the hip: a systematic review. Rheumatology (Oxford) 2002;41:1155-62.

17 Slavin RE. Best evidence synthesis: an intelligent alternative to meta-analysis. J Clin Epidemiol 1995;48:9-18.

18 Bieleman H, Reneman M, Drossaers-Bakker K, et al. Prognostic factors for sustained work participation in early osteoarthritis: a follow-up study in the Cohort Hip and Cohort Knee (CHECK). J Occup Rehabil 2013;23:74-81.

19 Bieleman HJ, Oosterveld FGJ, Oostveen JCM, et al. Work participation and health status in early osteoarthritis of the hip and/or knee: a comparison between the Cohort Hip and Cohort Knee and the Osteoarthritis Initiative. Arthritis Care Res 2010;62:683-9.

20 Andersen LL, Clausen T, Mortensen OS, et al. A prospective cohort study on musculoskeletal risk factors for long-term sickness absence among healthcare workers in eldercare. Int Arch Occup Environ Health 2012;85:615-22.

21 Andersen LL, Clausen T, Burr H, et al. Threshold of musculoskeletal pain intensity for increased risk of long-term sickness absence among female healthcare workers in eldercare. PLOS ONE 2012;7:e41287.

22 Leardini G, Salaffi F, Caporali R, et al. Direct and indirect costs of osteoarthritis of the knee. Clin Exp Rheumatol 2004;22:699-706.

23 Hubertsson J, Petersson IF, Thorstensson CA, et al. Risk of sick leave and disability pension in working-age women and men with knee osteoarthritis. Ann Rheum Dis 2013;72:401-5

24 Makela M, Heliovaara M, Sievers K, et al. Musculoskeletal disorders as determinants of disability in Finns aged 30 years or more. J Clin Epidemiol 1993:46:549-59.

25 Sayre EC, Li LC, Kopec JA, et al. The effect of disease site (knee, hip, hand, foot, lower back or neck) on employment reduction due to osteoarthritis. PLOS ONE 2010;5:e10470.

26 Woo J, Lau E, Lau CS, et al. Socioeconomic impact of osteoarthritis in Hong Kong: utilization of health and social services, and direct and indirect costs. Arthritis Rheum 2003;49:526-34.

27 Alexopoulos EC, Tanagra D, Detorakis I, et al. Knee and low back complaints in professional hospital nurses: occurrence, chronicity, care seeking and absenteeism. Work 2011;38:329-35.

28 Andersen LL, Mortensen OS, Hansen JV, et al. A prospective cohort study on severe pain as a risk factor for long-term sickness absence in blue- and white-collar workers. Occup Environ Med 2011;68:590-2.

29 Holmberg S, Stiernstrom E-L, Thelin A, et al. Musculoskeletal symptoms among farmers and non-farmers: a population-based study. Int J Occup Environ Health 2002;8:339-45

30 Merrill RM, Aldana SG, Pope JE, et al. Presenteeism according to healthy behaviors, physical health, and work environment. Popul Health Manag 2012; 15:293-301.

31 Morken T, Riise T, Moen B, et al. Low back pain and widespread pain predict sickness absence among industrial workers. BMC Musculoskelet Disord 2003;4:21.

32 Picavet HSJ, Schouten JSAG. Musculoskeletal pain in the Netherlands: prevalences, consequences and risk groups, the DMC3-study. Pain 2003;102:167-78.

33 Fautrel $B$, Hilliquin $P$, Rozenberg $S$, et al. Impact of osteoarthritis: results of a nationwide survey of 10,000 patients consulting for OA. Joint Bone Spine 2005; 72:235-40

34 Hutchings A, Calloway M, Choy E, et al. The Longitudinal Examination of Arthritis Pain (LEAP) study: relationships between weekly fluctuations in patient-rated joint pain and other health outcomes. J Rheumatol 2007; $34: 2291-300$. 
35 Lerner D, Amick BC III, Rogers WH, et al. The Work Limitations Questionnaire. Med Care 2001;39:72-85.

36 Kessler RC, Barber C, Beck A, et al. The World Health Organization Health and Work Performance Questionnaire (HPQ). J Occup Environ Med 2003;45:156-74.

37 Reilly MC, Zbrozek AS, Dukes EM. The validity and reproducibility of a work productivity and activity impairment instrument. Pharmacoeconomics 1993;4:353-65.

38 Goossens ME, Rutten-van Molken MP, Vlaeyen JW, et al. The cost diary: a method to measure direct and indirect costs in cost-effectiveness research. J Clin Epidemio/ 53:688-95.
39 Boers $\mathrm{M}$, Brooks $\mathrm{P}$, Simon $\mathrm{LS}$, et al. OMERACT: an international initiative to improve outcome measurement in rheumatology. Clin Exp Rheumatol 2005;23:S10-3.

40 Beaton D, Bombardier C, Escorpizo R, et al. Measuring worker productivity: frameworks and measures. J Rheumatol 2009;36:2100-9.

41 Ricci JA, Chee E. Lost productive time associated with excess weight in the U.S. workforce. J Occup Environ Med 47:1227-34.

42 Scheil-adlung $X$, Sandner L. Evidence on paid sick leave: Observations in times of crisis. Intereconomics 2010:45:313-21. 